\title{
EXPONENTIAL ENERGY DECAY FOR KERR-DE SITTER BLACK HOLES BEYOND EVENT HORIZONS
}

\author{
SEMYON DyATLOV
}

\begin{abstract}
We establish an exponential decay estimate for linear waves on the Kerr-de Sitter slowly rotating black hole. Combining the cutoff resolvent estimate of [10] with the red-shift effect and a parametrix near the event horizons, we obtain exponential decay on the whole domain of outer communications.
\end{abstract}

We study the decay of linear waves on the Kerr-de Sitter metric, corresponding to a rotating black hole in a spacetime with positive cosmological constant. (See, for example, [6] for the motivation for the problem and a survey of recent results.) Although in the original coordinates $(t, r, \theta, \varphi)$ the metric is only defined on $M=$ $\left\{r_{-}<r<r_{+}\right\}$and becomes singular on the event horizons $\left\{r=r_{ \pm}\right\}$, we will use a different coordinate system $\left(t_{+}, r, \theta, \varphi_{+}\right)$, in which the metric can be extended beyond the event horizons to $M_{\delta}=\left\{r_{-}-\delta<r<r_{+}+\delta\right\}$. In particular, $t_{+} \sim$ $t+C_{ \pm} \ln \left|r-r_{ \pm}\right|$near $r=r_{ \pm}$, with $C_{ \pm}$positive constants; see (1.4) for precise formulas and Section 1.2 in general for the description of the metric. The speed of rotation of the black hole is described by the parameter $a$; for $a=0$, we get the spherically symmetric Schwarzschild-de Sitter metric. We establish the following

Theorem 0.1. Let $\square_{g}$ be the d'Alembert-Beltrami operator of the Kerr-de Sitter metric on $M_{\delta}$. Fix $\varkappa>0$. For $a, \delta>0, \nu>0$ small enough and any $s \geq 1$, there exists a constant $C$ such that if $u \in H_{\mathrm{loc}}^{s+\varkappa+1}\left(M_{\delta}\right)$ is a solution to the equation $\square_{g} u=f \in H_{\mathrm{loc}}^{s+\varkappa}\left(M_{\delta}\right)$, with $\operatorname{supp} u \subset\left\{t_{+}>-T\right\}$ for some $T$, then

$$
\left\|e^{\nu t_{+}}\left(u-\Pi_{0} f\right)\right\|_{H^{s}\left(M_{\delta}\right)} \leq C\left\|e^{\nu t_{+}} f\right\|_{H^{s+\varkappa}\left(M_{\delta}\right)} .
$$

Here

$$
\Pi_{0} f=\frac{1+\alpha}{4 \pi\left(r_{+}^{2}+r_{-}^{2}+2 a^{2}\right)} \int_{M} f d \mathrm{Vol}
$$

is a constant (note that we integrate over $M$, not the whole $M_{\delta}$ ); $H^{s}$ norms are taken with respect to the $\left(t_{+}, r, \theta, \varphi_{+}\right)$coordinates (here $\left(\theta, \varphi_{+}\right)$are treated as spherical coordinates on $\mathbb{S}^{2}$ ).

The main ingredient of the proof, which gives us exponential decay, is the scattering resolvent constructed in [10]. We modify the argument of [10, Theorem 6] to get exponential decay for $u$ on a certain compact subset $K_{\delta} \subset M$, under the condition that $f$ is supported in $K_{\delta}$ as well (Proposition 2.1). In the present paper, we specify $\square_{g} u$, rather than the Cauchy data of $u$, to facilitate the proofs. However, it is not hard to convert Theorem 0.1 to an exponential decay estimate for the Cauchy problem, such as the one given in [10].

Received by the editors October 26, 2010. 
The second ingredient, described in Section 1.3, is the energy estimate produced by the red-shift effect at the event horizons, first introduced by Dafermos and Rodnianski in $[4,7,8]$. Paper [4] in particular introduced the idea that the red-shift implies that boundedness and decay properties propagate from the event horizon to a neighborhood of it in the black hole interior. The vector field approach to the red-shift effect was introduced in [7]; in [8], the method was extended to higher-order estimates using the remark that commutation generated further terms of favorable sign. Paper [7] established red-shift estimates for the Schwarzschild black hole, while [8] considered the case of slowly rotating Kerr; the (subextremal) Kerr-de Sitter horizons are in particular covered by [6, Theorems 7.1 and 7.2]. It should also be noted that in certain cases, such as extremal Reissner-Nordström spacetimes considered by Aretakis $[1,2]$, the failure of the red-shift is directly related to instabilities of linear waves at the event horizon.

In our presentation, we follow both [6, Section 3.3] and the paper [17] by Tataru and Tohaneanu on integrated decay for the Kerr black hole. Combining the red-shift effect with Proposition 2.1, we obtain an estimate on $u$ on the whole $M_{\delta}$, provided that $f$ is still supported in $K_{\delta}$. Finally, we use a Morawetz-type argument together with red-shift (Proposition 2.3) to construct an exponentially decaying parametrix for the wave equation near the event horizons and reduce the general problem to the case supp $f \subset K_{\delta}$.

Compared to the energy estimate for the Minkowski spacetime (Proposition 1.4), we lose $1+\varkappa$ derivatives in Theorem 1 , where $\varkappa>0$ can be arbitrarily small. This is related to the exponent in the polynomial resolvent estimate of [10], which in turn is determined by the separation of variables procedure employed there [10, Proposition 3.4]. It is possible that a more careful analysis will yield a smaller loss in derivatives; however, the presence of trapping indicates that loss of regularity is inevitable (see [15] for a precise statement in the now classical case of obstacle scattering).

Exponential decay of linear waves on the Schwarzschild-de Sitter metric has been studied in [3, 5, 14]. Dafermos and Rodnianski [5], using vector field multipliers, proved that linear waves decay faster than every negative power of $t_{+}$. Bony and Häfner [3], building on earlier work on the scattering resolvent by Sá Barreto and Zworski [16], showed exponential decay away from the event horizons. Finally, Melrose et al. [14] proved exponential decay up to the event horizons. The latter result, combined with the recent work on normally hyperbolic trapping [19] and gluing semiclassical resolvent estimates [9], can be applied to certain short-range stationary perturbations of the Schwarzschild-de Sitter spacetime; see [9, Corollary 6.1]. It should be noted, however, that Kerr-de Sitter is not an acceptable perturbation, in particular because the theorem of Mazzeo and Melrose [13] does not apply to the low-energy situation anymore. Therefore, at the moment, the results of [10] seem necessary for obtaining exponential decay of waves on Kerr-de Sitter.

\section{Kerr-de Sitter metric and the red-shift effect}

1.1. Energy estimates. We recall some well-known facts from Lorentzian geometry; see, for example, [6, Appendices] or [18, Section 2.8] for a more detailed account.

Let $M$ be an $n$-dimensional smooth manifold and $g$ be a Lorentzian metric; that is, a symmetric $(0,2)$-tensor $g$ of signature $(1, n-1)$. (Sometimes a different convention 
is used, in which the metric has signature $(n-1,1)$.) The basic example is the space $\mathbb{R}_{t, x}^{n}$ with the Minkowski metric

$$
d t^{2}-\sum_{j=1}^{n-1} d x_{j}^{2}
$$

A tangent vector $X$ is called timelike if $g(X, X)>0$, null if $g(X, X)=0$, and spacelike if $g(X, X)>0$. If $X$ and $Y$ are two timelike vectors, then we say that they point in the same direction if $g(X, Y)>0$ and they point in opposite directions if $g(X, Y)<0$. This definition can be extended to cases when $X$ and/or $Y$ is a nonzero null vector. A hyperplane in a tangent space is called spacelike if its normal vector (with respect to $g$ ) is timelike, timelike if its normal vector is spacelike, and null if its normal vector is null. Note that the restriction of $g$ to a spacelike hyperplane is negative definite, while the restriction to a null plane has signature $(0, n-2)$.

We now describe a way of obtaining energy estimates for the wave equation on Lorentzian manifolds. Let $\Omega \subset M$ be a bounded domain and $u \in C^{\infty}(\bar{\Omega})$. Define the symmetric $(0,2)$-tensor $T_{\nabla u}$ by the formula

$$
T_{\nabla u}(X, Y)=(X u)(Y u)-\frac{1}{2} g(\nabla u, \nabla u) g(X, Y),
$$

valid for all vector fields $X, Y$ on $\Omega$. Note that for fixed $X$ and $Y, T_{\nabla u}(X, Y)$ is a quadratic form in $\nabla u$. If $X$ and $Y$ are both timelike, then this form is positive definite in $\nabla u$ for $X$ and $Y$ pointing in the same direction and negative definite otherwise. Same is true if $X$ and/or $Y$ is null, with the form being nonnegative or nonpositive, respectively.

Fix a vector field $X$ on $\Omega$ and consider the vector field $J_{X}(u)$, given by the formula

$$
g\left(J_{X}(u), Y\right)=T_{\nabla u}(X, Y),
$$

valid for all vector fields $Y$. The divergence theorem then gives

$$
\int_{\partial \Omega} T_{\nabla u}(X, \vec{n}) d S=\int_{\Omega} \operatorname{div} J_{X}(u) d \mathrm{Vol} .
$$

Here $\vec{n}$ is the unit normal vector pointing outward (in the sense that $g(\vec{n}, Z)>0$ for every vector $Z$ pointing outside of $\Omega$ ); $d S$ is the area measure induced by the restriction of $g$ to $\partial \Omega$, and $d \mathrm{Vol}$ is the volume measure induced by $g$. One has to take care when defining the left-hand side of (1.2) at the points where $\partial \Omega$ is null, as $\vec{n}$ blows up, being both unit and null, and $d S$ is equal to zero; see [6, Appendix $\mathrm{C}]$ for details. The discussion following (1.1) implies

Proposition 1.1. Let $\mathcal{C}$ be an open subset of $\partial \Omega$ whose tangent space is either spacelike or null at every point. Moreover, assume that $X$ is timelike and points outside of $\Omega$ on $\mathcal{C}$. Then for every $u$,

$$
\int_{\mathcal{C}} T_{\nabla u}(X, \vec{n}) d S \geq 0
$$

The sign of the flux of $J_{X}$ over a timelike piece of $\partial \Omega$ cannot be determined in general; however, we can find it if $u$ satisfies a boundary condition:

Proposition 1.2. Let $\mathcal{C}$ be an open timelike subset of $\partial \Omega$ and assume that $\left.u\right|_{\mathcal{C}}=0$. If $X$ points inside of $\Omega$ on $\mathcal{C}$, then (1.3) holds. 
Proof. We have $\nabla u=v \vec{n}$ on $\mathcal{C}$, for some function $v$. Then

$$
T_{\nabla u}(X, \vec{n})=\frac{v^{2}}{2} g(X, \vec{n}) g(\vec{n}, \vec{n})=-\frac{v^{2}}{2} g(X, \vec{n}) \geq 0 .
$$

Finally, we relate the divergence of $J_{X}$ to the d'Alembert-Beltrami operator $\square_{g}$ :

Proposition 1.3. Let $\mathcal{L}_{X} g$ be the Lie derivative of $g$ with respect to $X$, and consider the symmetric $(0,2)$-tensor $K^{X}$ given by

$$
K^{X}=\frac{1}{2} \mathcal{L}_{X} g-\frac{1}{4} \operatorname{Tr}\left(g^{-1} \mathcal{L}_{X} g\right) g .
$$

Then

$$
\operatorname{div} J_{X}(u)=(X u) \square_{g} u+K^{X}(\nabla u, \nabla u) .
$$

As a basic application, we prove the energy estimate for the constant-coefficient wave equation:

Proposition 1.4. Fix $0<T<R$ and consider the domain

$$
\Omega=\{0<t<T,|x|<R-t\}
$$

in the Minkowski spacetime. Let $u \in C^{\infty}(\bar{\Omega})$ and define the energy

$$
E(s)=\frac{1}{2} \int_{\substack{t=s \\|x|<R-s}}\left|u_{t}\right|^{2}+\left|\partial_{x} u\right|^{2} d x, \quad 0 \leq s \leq T .
$$

Then

$$
E(T) \leq E(0)+\int_{\Omega} u_{t} \square u d t d x .
$$

Proof. We take $X=\partial_{t}$ and apply (1.2) on $\Omega$. Since $X$ is Killing, $K^{X}=0$ and thus

$$
\int_{\partial \Omega} T_{\nabla u}(X, \vec{n}) d S=\int_{\Omega} u_{t} \square u d t d x .
$$

Now, the boundary of $\Omega$ consists of the following pieces:

$$
\begin{gathered}
\mathcal{P}_{0}=\{t=0,|x|<R\}, \\
\mathcal{P}_{T}=\{t=T,|x|<R-T\}, \\
\mathcal{C}=\{0 \leq t \leq T,|x|=R-t\} .
\end{gathered}
$$

The integral over $\mathcal{P}_{0}$ is equal to $-E(0)$ and the integral over $\mathcal{P}_{T}$ is equal to $E(T)$. Finally, the integral over $\mathcal{C}$ is nonnegative by Proposition 1.1, as $\mathcal{C}$ is null and $\partial_{t}$ points outside of $\Omega$ on $\mathcal{C}$.

1.2. Kerr-de Sitter metric. The Kerr-de Sitter metric is given by

$$
\begin{aligned}
g= & -\rho^{2}\left(\frac{d r^{2}}{\Delta_{r}}+\frac{d \theta^{2}}{\Delta_{\theta}}\right) \\
& -\frac{\Delta_{\theta} \sin ^{2} \theta}{(1+\alpha)^{2} \rho^{2}}\left(a d t-\left(r^{2}+a^{2}\right) d \varphi\right)^{2} \\
& +\frac{\Delta_{r}}{(1+\alpha)^{2} \rho^{2}}\left(d t-a \sin ^{2} \theta d \varphi\right)^{2}
\end{aligned}
$$


Here $M_{0}$ is the mass of the black hole, $\Lambda$ is the cosmological constant (both of which we assume to be fixed), and $a M_{0}$ is the angular momentum (which we assume to be small);

$$
\begin{gathered}
\Delta_{r}=\left(r^{2}+a^{2}\right)\left(1-\frac{\Lambda r^{2}}{3}\right)-2 M_{0} r, \\
\Delta_{\theta}=1+\alpha \cos ^{2} \theta, \\
\rho^{2}=r^{2}+a^{2} \cos ^{2} \theta, \alpha=\frac{\Lambda a^{2}}{3} .
\end{gathered}
$$

The metric in the $(t, r, \theta, \varphi)$ coordinates is defined for $\Delta_{r}>0$; we assume that $r_{ \pm}$ are two positive roots of the equation $\Delta_{r}=0$, such that $\Delta_{r}>0$ on the open interval $0<r_{-}<r<r_{+}<\infty$. The variables $\theta \in[0, \pi]$ and $\varphi \in \mathbb{R} / 2 \pi \mathbb{Z}$ are the spherical coordinates on the sphere $\mathbb{S}^{2}$. The spacetime is then

$$
M=\mathbb{R}_{t} \times\left(r_{-}, r_{+}\right) \times \mathbb{S}_{\theta, \varphi}^{2}
$$

(Note the difference in notation with [10].) The volume form is

$$
d \mathrm{Vol}=\frac{\rho^{2} \sin \theta}{(1+\alpha)^{2}} d t d r d \theta d \varphi
$$

For $a=0$, we get the Schwarzschild-de Sitter metric:

$$
g_{0}=-\frac{r^{2}}{\Delta_{r}} d r^{2}+\frac{\Delta_{r}}{r^{2}} d t^{2}-r^{2} g_{S}
$$

where

$$
g_{S}=d \theta^{2}+\sin ^{2} \theta d \varphi^{2}
$$

is the round metric on the sphere of radius 1 .

Next, we introduce a modification of the Kerr-star coordinates (see [6, Section 5.1]). We remove the singularities at $r=r_{ \pm}$by making the change of variables $(t, r, \theta, \varphi) \rightarrow$ $\left(t_{+}, r, \theta, \varphi_{+}\right)$, where

$$
t_{+}=t-F_{t}(r), \varphi_{+}=\varphi-F_{\varphi}(r) .
$$

Note that $\partial_{t_{+}}=\partial_{t}$ and $\partial_{\varphi_{+}}=\partial_{\varphi}$. The functions $F_{t}$ and $F_{\varphi}$ are required to be smooth on $\left(r_{-}, r_{+}\right)$and satisfy the following condition:

$$
F_{t}^{\prime}(r)= \pm \frac{(1+\alpha)\left(r^{2}+a^{2}\right)}{\Delta_{r}}, F_{\varphi}^{\prime}(r)= \pm \frac{(1+\alpha) a}{\Delta_{r}} \text { for }\left|r-r_{ \pm}\right|<\varepsilon
$$

Here $\varepsilon>0$ is some fixed small constant. The metric $g$ in the $\left(t_{+}, r, \theta, \varphi_{+}\right)$coordinates takes the following form for $\left|r-r_{ \pm}\right|<\delta$ :

$$
\begin{gathered}
-\rho^{2} \frac{d \theta^{2}}{\Delta_{\theta}}-\frac{\Delta_{\theta} \sin ^{2} \theta}{(1+\alpha)^{2} \rho^{2}}\left(a d t_{+}-\left(r^{2}+a^{2}\right) d \varphi_{+}\right)^{2} \\
+\frac{\Delta_{r}}{(1+\alpha)^{2} \rho^{2}}\left(d t_{+}-a \sin ^{2} \theta d \varphi_{+}\right)^{2} \pm \frac{2}{(1+\alpha)}\left(d t_{+}-a \sin ^{2} \theta d \varphi_{+}\right) d r .
\end{gathered}
$$


We see that the metric is smooth up to the event horizons $\left\{r=r_{ \pm}\right\}$; moreover, for $\delta$ small enough, we can extend it to

$$
M_{\delta}=\left\{r_{-}-\delta \leq r \leq r_{+}+\delta\right\} .
$$

The event horizons are null, while the surfaces $\left\{r=r_{0}\right\}$ are spacelike for $r_{0} \notin\left[r_{-}, r_{+}\right]$. The time surfaces $\left\{t_{+}=\right.$const $\}$are null near the event horizons; however, one can shift the time variable a little bit (see [10, Section 1]) to make the problem

$$
\square_{g} u=f \in C_{0}^{\infty}\left(M_{\delta}\right), \quad \operatorname{supp} u \subset\left\{t_{+}>-T\right\} \text { for some } T
$$

well posed. We call $u$ the forward solution of the equation $\square_{g} u=f$.

Finally, note that the field $\partial_{t}$ (which is the same in the $(t, r, \theta, \varphi)$ and $\left(t_{+}, r, \theta, \varphi_{+}\right)$ coordinates) is not timelike on $M$ inside the two surfaces located $O(a)$-close (in the $r$ variable) to the event horizons; these surfaces are called the ergospheres.

1.3. Red-shift effect. In this section, we prove the following energy estimate:

Proposition 1.5. For $\delta>0$, define

$$
K_{\delta}=\left\{r_{-}+\delta<r<r_{+}-\delta\right\} \subset M .
$$

Then for $\delta, a$, and $\nu>0$ small enough, $s$ a nonnegative integer, and every forward solution $u$ to the equation $\square_{g} u=f \in C_{0}^{\infty}\left(M_{\delta}\right)$, we have ${ }^{1}$

$$
\left\|e^{\nu t_{+}} u\right\|_{H^{s+1}\left(M_{\delta}\right)} \lesssim\left\|e^{\nu t_{+}} f\right\|_{H^{s}\left(M_{\delta}\right)}+\left\|e^{\nu t} u\right\|_{H^{s+1}\left(K_{\delta}\right)} .
$$

We start the proof with the construction of a special vector field; see also $[6$, Proposition 3.3.1].

Proposition 1.6. For $\delta>0$ and a small enough, there exists a vector field $X$ defined on $M_{\delta} \backslash K_{2 \delta}$, with the following properties:

- $X$ is stationary; that is, $\left[X, \partial_{t}\right]=0$.

- $X$ is timelike and $X t_{+}>0, \pm X r>0$ on $M_{\delta} \backslash K_{2 \delta}$.

- The tensor $K^{X}$, defined in Proposition 1.3, is negative definite on $M_{\delta} \backslash K_{2 \delta}$.

Proof. We will construct $X$ for $a=0$; same field will work for small $a$ since the components of the Kerr-de Sitter metric near the event horizons are continuous functions of $a$. Moreover, since $\delta>0$ can be chosen arbitrarily small, we only need to verify properties of $X$ at the event horizons. We use the $\left(t_{+}, r, \theta, \varphi_{+}\right)$coordinates. The metric for $a=0$ has the form

$$
\frac{\Delta_{r}}{r^{2}} d t_{+}^{2} \pm 2 d t_{+} d r-r^{2} g_{S}
$$

for $\left|r-r_{ \pm}\right|<2 \delta$; if we take

$$
X=X_{r}(r) \partial_{r}+X_{t}(r) \partial_{t}
$$

\footnotetext{
${ }^{1}$ We write $(A) \lesssim(B)$, if there exists some constant $C$, independent of the choice of $f$, such that $A \leq C(B)$. Here $C$ might depend on the parameters of the problem such as $\nu, s$, and $\varkappa$.
} 
where $X_{r}, X_{t}$ are some functions, then at $r=r_{ \pm}$,

$$
\begin{gathered}
\mathcal{L}_{X} g=X_{r}\left(\frac{\Delta_{r}^{\prime}}{r^{2}} d t_{+}^{2}-2 r g_{S}\right) \pm 2\left[\partial_{r} X_{t} d r^{2}+\partial_{r} X_{r} d r d t_{+}\right] \\
K^{X}=\frac{X_{r} \Delta_{r}^{\prime}}{2 r^{2}} d t_{+}^{2} \pm \partial_{r} X_{t} d r^{2} \mp \frac{2 X_{r}}{r} d r d t_{+}+\frac{r^{2}}{2} \partial_{r} X_{r} g_{S}
\end{gathered}
$$

We put $X_{t}=1$ and $X_{r}= \pm 1$ at $r=r_{ \pm}$; then the field $X$ is timelike and $d t_{+}(X)>0$. To make $K^{X}$ negative definite, it then suffices to take $\mp \partial_{r} X_{t}$ positive and large enough and $\partial_{r} X_{r}$ negative at the event horizons.

Remark. Note that the only component of $K^{X}$ whose sign is definite independently of the choice of $\partial_{r} X$ is

$$
K^{X}\left(\partial_{t}, \partial_{t}\right)=\frac{1}{2}\left(L_{x} g\right)_{t_{+} t_{+}}=-g\left(X, \nabla_{\partial_{t}} \partial_{t}\right)
$$

One can compute

$$
\nabla_{\partial_{t}} \partial_{t}=\kappa \partial_{t}
$$

for some constant $\kappa>0$; then,

$$
K^{X}\left(\partial_{t}, \partial_{t}\right)=\mp \kappa X_{r}, r=r_{ \pm}
$$

Equation (1.6) can be interpreted as follows: the momentum is exponentially decaying as a function of the geodesic parameter on the family of trapped geodesics $\{r=$ $r_{ \pm},(\theta, \varphi)=$ const $\}$. This is related to the classical red-shift effect; see [6, Sections 3.3.2 and 7.1] for more details.

We are now ready to prove Proposition 1.5. To facilitate the inductive argument for estimating higher derivatives, we show the following more general fact:

Proposition 1.7. Assume that $\psi(r)$ is a function on $M_{\delta}$ such that $\psi \geq 0$ outside of $K_{\delta}$, and $u$ is a forward solution to the equation

$$
\left(\square_{g}+\psi X\right) u=f \in C_{0}^{\infty}\left(M_{\delta}\right) .
$$

Here $X$ is the field constructed in Proposition 1.6. Then for $a, \delta>0, \nu>0$ small enough and each nonnegative integer $s$,

$$
\left\|e^{\nu t_{+}} u\right\|_{H^{s+1}\left(M_{\delta}\right)} \lesssim\left\|e^{\nu t_{+}} f\right\|_{H^{s}\left(M_{\delta}\right)}+\left\|e^{\nu t_{+}} u\right\|_{H^{s+1}\left(K_{\delta}\right)} .
$$

Proof. We use induction on $s$. First, assume that $s=0$. Take a nonnegative function $\chi(r)$ on $M_{\delta}$ such that $\chi=0$ near $K_{2 \delta}$, but $\chi=1$ away from $K_{\delta}$. Let $T>0$ and apply the divergence theorem in the region

$$
\Omega_{T}=M_{\delta} \cap\left\{t_{+}<T\right\}
$$

to the vector field

$$
V=e^{2 \nu t_{+}} \chi J_{X}(u)
$$

Here $J_{X}$ is defined in Section 1.1. (The divergence theorem holds, despite $\Omega_{T}$ being noncompact, since $u$ is a forward solution.) We compute by Proposition 1.3

$$
\begin{aligned}
\operatorname{div} V= & e^{2 \nu t_{+}}\left[2 \nu \chi d t_{+}\left(J_{X}(u)\right)+e^{2 \nu t_{+}} \chi^{\prime} d r\left(J_{X}(u)\right)\right. \\
& \left.+\chi(X u) f-\chi \psi(X u)^{2}+\chi K^{X}(\nabla u, \nabla u)\right]
\end{aligned}
$$


The flux of $V$ is nonnegative by Proposition 1.1; therefore, by letting $T \rightarrow+\infty$ we get

$$
\begin{aligned}
& \int_{M_{\delta} \backslash K_{\delta}}-e^{2 \nu t_{+}} K^{X}(\nabla u, \nabla u) d \mathrm{Vol} \lesssim \nu\left\|e^{\nu t_{+}} u\right\|_{H^{1}\left(M_{\delta}\right)}^{2} \\
& \quad+\left\|e^{\nu t_{+}} u\right\|_{H^{1}\left(K_{\delta}\right)}^{2}+\left\|e^{\nu t_{+}} u\right\|_{H^{1}\left(M_{\delta}\right)} \cdot\|f\|_{L^{2}\left(M_{\delta}\right)}
\end{aligned}
$$

Since $K^{X}$ is negative definite on $M_{\delta} \backslash K_{\delta}$ and by Poincaré inequality, we have for $\nu$ small enough,

$$
\left\|e^{\nu t_{+}} u\right\|_{H^{1}\left(M_{\delta}\right)}^{2} \lesssim\left\|e^{\nu t_{+}} u\right\|_{H^{1}\left(K_{\delta}\right)}^{2}+\left\|e^{\nu t_{+}} u\right\|_{H^{1}\left(M_{\delta}\right)} \cdot\|f\|_{L^{2}\left(M_{\delta}\right)}
$$

This finishes the proof of (1.7) for $s=0$.

Now, assume that $s \geq 1$ and (1.7) is true for $s-1$; we will prove it for $s$ following [8, Sections 1.7.5 and 10] (see also [17, Theorem 4.4]). First, let $Y$ be equal to either $\partial_{t}$ or a Killing field on $\mathbb{S}^{2}$; then $[\psi X, Y]=0$ and, since the metric is spherically symmetric for $a=0,\left[\square_{g}, Y\right]$ is a second-order differential operator with $O(a)$ coefficients. We have

$$
\left(\square_{g}+\psi X\right) Y u=Y f+\left[\square_{g}, Y\right] u
$$

therefore, by (1.7),

$$
\left\|e^{\nu t_{+}} Y u\right\|_{H^{s}\left(M_{\delta}\right)} \lesssim\left\|e^{\nu t_{+}} Y f\right\|_{H^{s-1}\left(M_{\delta}\right)}+O(a)\left\|e^{\nu t_{+}} u\right\|_{H^{s+1}\left(M_{\delta}\right)}+\left\|e^{\nu t_{+}} Y u\right\|_{H^{s}\left(K_{\delta}\right)} .
$$

Therefore, if $\hat{\partial} u$ is composed of derivatives of $u$ with respect to $t_{+}, \theta, \varphi_{+}$, then

$$
\left\|e^{\nu t_{+}} \hat{\partial} u\right\|_{H^{s}\left(M_{\delta}\right)} \lesssim\left\|e^{\nu t_{+}} f\right\|_{H^{s}\left(M_{\delta}\right)}+O(a)\left\|e^{\nu t_{+}} u\right\|_{H^{s+1}\left(M_{\delta}\right)}+\left\|e^{\nu t_{+}} u\right\|_{H^{s+1}\left(K_{\delta}\right)} .
$$

Now, we estimate $\partial_{r} u$. We can write

$$
\left[\square_{g}+\psi X, \partial_{r}\right]=-\eta X \partial_{r}+L
$$

where $L$ is a second-order differential operator not containing any $\partial_{r}^{2}$ terms and $\eta$ is positive near the event horizons. Then

$$
\left\|e^{\nu t_{+}} L u\right\|_{H^{s-1}\left(M_{\delta}\right)} \lesssim\left\|e^{\nu t_{+}} \hat{\partial} u\right\|_{H^{s}\left(M_{\delta}\right)}+\left\|e^{\nu t_{+}} u\right\|_{H^{s}\left(M_{\delta}\right)} .
$$

We have

$$
\left(\square_{g}+(\psi+\eta) X\right) \partial_{r} u=\partial_{r} f+L u ;
$$

since $\psi+\eta \geq 0$ near the event horizons, by (1.7) applied to $\partial_{r} u$ and (1.8) we get

$$
\begin{aligned}
& \left\|e^{\nu t_{+}} u\right\|_{H^{s+1}\left(M_{\delta}\right)} \lesssim\left\|e^{\nu t_{+}} \partial_{r} u\right\|_{H^{s}\left(M_{\delta}\right)}+\left\|e^{\nu t_{+}} \hat{\partial} u\right\|_{H^{s}\left(M_{\delta}\right)} \\
& \lesssim\left\|e^{\nu t_{+}} f\right\|_{H^{s}\left(M_{\delta}\right)}+\left\|e^{\nu t_{+}} u\right\|_{H^{s+1}\left(K_{\delta}\right)}+\left\|e^{\nu t_{+}} \hat{\partial} u\right\|_{H^{s}\left(M_{\delta}\right)} \\
& +\left\|e^{\nu t_{+}} u\right\|_{H^{s}\left(M_{\delta}\right)} \\
& \lesssim\left\|e^{\nu t_{+}} f\right\|_{H^{s}\left(M_{\delta}\right)}+\left\|e^{\nu t_{+}} u\right\|_{H^{s+1}\left(K_{\delta}\right)}+O(a)\left\|e^{\nu t_{+}} u\right\|_{H^{s+1}\left(M_{\delta}\right)} \text {; }
\end{aligned}
$$

it remains to take $a$ small enough. 


\section{Proof of exponential decay}

Throughout this section, $u$ is a forward solution to the equation $\square_{g} u=f$, with $f \in$ $C_{0}^{\infty}\left(M_{\delta}\right)$. (The estimates for general $f$ can then be obtained by a density argument.)

2.1. Case of $f$ supported in $K_{\delta}$. First of all, we use the resolvent estimates of [10] to obtain exponential decay away from the event horizons:

Proposition 2.1. Fix $\delta>0, \varkappa>0$ and assume that $\chi(r) \in C_{0}^{\infty}\left(r_{-}+\delta, r_{+}-\delta\right)$. Then for a small enough and every $s \geq 0$, we have

$$
\left\|e^{\nu t} \chi(r)\left(u-\Pi_{0} f\right)\right\|_{H^{s}} \lesssim\left\|e^{\nu t} f\right\|_{H^{s+\varkappa}}
$$

for every $f \in C_{0}^{\infty}\left(K_{\delta}\right)$. (We can use $e^{\nu t}$ in place of $e^{\nu t_{+}}$, as $\left|t-t_{+}\right|$is bounded and the two weights are equivalent in $K_{\delta}$.)

Proof. We use the argument of [10, Theorem 6]. By [10, Proposition 1.1], $e^{-C t} u$ is tempered in the time variable for some constant $C$; therefore, the Fourier-Laplace transform

$$
\hat{u}(\omega, \cdot)=\int_{-\infty}^{\infty} e^{i t \omega} u(t, \cdot) d t
$$

is well defined and holomorphic in $\{\operatorname{Im} \omega>C\}$. Let $K_{S}=\left(r_{-}+\delta, r_{+}-\delta\right) \times \mathbb{S}^{2}$ be the space slice of $K_{\delta}$. We choose $a$ small enough so that [10, Theorem 2] provides us with the scattering resolvent $R_{g}(\omega): L^{2}\left(K_{S}\right) \rightarrow L^{2}\left(K_{S}\right)$; it is a family of operators meromorphic in the entire complex plane. By [10, Proposition 1.2],

$$
\chi(r) \hat{u}(\omega)=\chi(r) R_{g}(\omega)\left(\rho^{2} \hat{f}(\omega)\right),
$$

where $\rho(r, \theta)$ is the smooth function defined in Section 1.2 and $\hat{f}(\omega)$ is an entire function that is rapidly decaying in $\omega$ for $\operatorname{Im} \omega$ bounded, with values in $C_{0}^{\infty}\left(K_{S}\right)$. Now, there exists $\nu>0$ such that $R_{g}(\omega)$ is holomorphic and polynomially bounded in $\{\operatorname{Im} \omega \geq-\nu\}$, except for a pole at zero [10, Theorems 4 and 5]. Therefore, we can use Fourier inversion formula and contour deformation to get

$$
\chi(r)\left(u(t, \cdot)-\Pi_{0} f\right)=\frac{1}{2 \pi} \int_{\operatorname{Im} \omega=-\nu} e^{-i t \omega} \chi(r) R_{g}(\omega)\left(\rho^{2} \hat{f}(\omega)\right) d \omega
$$

the residue at zero being exactly $\Pi_{0} f$. Now, let $s \in \mathbb{R}$, put $h=\langle\omega\rangle^{-1}$, and introduce the semiclassical Sobolev space $H_{h, \text { comp }}^{s}\left(K_{S}\right) \subset \mathcal{E}^{\prime}\left(K_{S}\right)$; the norm of $v \in \mathcal{E}^{\prime}\left(K_{S}\right)$ in this space is given by $\left\|\langle h D\rangle^{s} v\right\|_{L^{2}}$, where $\langle h D\rangle^{s}$ is a Fourier multiplier and $v$ is extended by zero to $\mathbb{R}^{3} \supset K_{S}$. Then the norm of $e^{\nu t} f$ in $H^{s}\left(K_{\delta}\right)$ is equivalent to the norm of $\langle\omega\rangle^{s} \hat{f}$ in $L_{\nu}^{2} H_{h}^{s}$, where

$$
\|v\|_{L_{\nu}^{2} H_{h}^{s}}^{2}=\int_{\operatorname{Im} \omega=-\nu}\|v(\omega)\|_{H_{h}^{s}}^{2} d \omega .
$$

This, together with the resolvent estimate of [10, Theorem 5] gives for $\varkappa$ fixed and $\nu$ small enough,

$$
\left\|\langle\omega\rangle^{s} \chi(r) R_{g}(\omega)\left(\rho^{2} \hat{f}(\omega)\right)\right\|_{L_{\nu}^{2} H_{h}^{0}} \lesssim\left\|e^{\nu t} f\right\|_{H^{s+\varkappa}}
$$


Now, we use that $R_{g}(\omega)$ is a right inverse to the second-order differential operator $[10$, Section 1]

$$
\begin{aligned}
P_{g}(\omega)= & D_{r}\left(\Delta_{r} D_{r}\right)-\frac{(1+\alpha)^{2}}{\Delta_{r}}\left(\left(r^{2}+a^{2}\right) \omega-a D_{\varphi}\right)^{2} \\
& +\frac{1}{\sin \theta} D_{\theta}\left(\Delta_{\theta} \sin \theta D_{\theta}\right)+\frac{(1+\alpha)^{2}}{\Delta_{\theta} \sin ^{2} \theta}\left(a \omega \sin ^{2} \theta-D_{\varphi}\right)^{2}
\end{aligned}
$$

then $h^{2} P_{g}(\omega)$ is a semiclassical pseudodifferential operator and for $a$ small enough, it is elliptic on $K_{S}$ outside of some $\omega$-independent compact set. (This is equivalent to saying that $K_{\delta}$ does not intersect the ergosphere.) We can construct a semiclassical parametrix (see, for example, [11, Section 4.5] or [10, Proposition 5.1]); i.e., a properly supported semiclassical pseudodifferential operator on $Q$ on $K_{S}$ that maps $H_{h, \text { loc }}^{s}\left(K_{S}\right) \rightarrow H_{h, \text { loc }}^{s+2}\left(K_{S}\right)$ for all $s$ and such that $I-Q h^{2} P_{g}(\omega) \operatorname{maps} H_{h, \text { loc }}^{-N}\left(K_{S}\right) \rightarrow$ $H_{h, \text { loc }}^{N}\left(K_{S}\right)$ with norm $O(1)$ for all $N$. Then for any $\chi_{1}(r) \in C_{0}^{\infty}\left(r_{-}, r_{+}\right)$that is nonzero near supp $\chi$ and any $v \in C_{0}^{\infty}\left(K_{S}\right)$, we can apply $I-Q h^{2} P_{g}(\omega)$ to $R_{g}(\omega) v$ to get

$$
\left\|\chi(r) R_{g}(\omega) v\right\|_{H_{h}^{s}} \lesssim\langle\omega\rangle^{-2}\|v\|_{H_{h}^{s-2}}+\left\|\chi_{1}(r) R_{g}(\omega) v\right\|_{H_{h}^{0}}
$$

Therefore,

$$
\left\|\langle\omega\rangle^{s} \chi(r) R_{g}(\omega)\left(\rho^{2} \hat{f}(\omega)\right)\right\|_{L_{\nu}^{2} H_{h}^{s}} \lesssim\left\|e^{\nu t} f\right\|_{H^{s+\varkappa}}
$$

it remains to combine this with (2.1).

Combining the above fact with the red-shift estimate, we get

Proposition 2.2. Fix $\delta>0$ such that Proposition 1.5 holds and choose a small enough so that Proposition 2.1 holds for $\delta / 2$ in place of $\delta$. Take $\nu>0$ small enough so that both propositions above hold. Then for $s \geq 1$ and every $\varkappa>0$, we have

$$
\left\|e^{\nu t_{+}}\left(u-\Pi_{0} f\right)\right\|_{H^{s}\left(M_{\delta}\right)} \lesssim\left\|e^{\nu t} f\right\|_{H^{s+\varkappa}\left(K_{\delta}\right)},
$$

for every $f \in C_{0}^{\infty}\left(K_{\delta}\right)$.

Proof. We consider the case of integer $s$; the general case follows by interpolation in Sobolev spaces (see, for example, [18, Section 4.2]). Let $\psi\left(t_{+}\right)$be a smooth function that is equal to 1 for $t_{+}$large positive and to 0 for $t_{+}$large negative; take large $T \in \mathbb{R}$ and apply Proposition 1.5 to $u-\psi\left(t_{+}+T\right) \Pi_{0} f$ :

$$
\begin{aligned}
& \left\|e^{\nu t_{+}}\left(u-\Pi_{0} f\right)\right\|_{H^{s}\left(M_{\delta}\right)} \\
& \quad \lesssim\left\|e^{\nu t_{+}}\left(u-\psi\left(t_{+}+T\right) \Pi_{0} f\right)\right\|_{H^{s}\left(M_{\delta}\right)}+\left\|e^{\nu t_{+}}\left(1-\psi\left(t_{+}+T\right)\right) \Pi_{0} f\right\|_{H^{s}\left(M_{\delta}\right)} \\
& \quad \lesssim\left\|e^{\nu t_{+}} f\right\|_{H^{s-1}\left(M_{\delta}\right)}+\left\|e^{\nu t_{+}}\left(u-\Pi_{0} f\right)\right\|_{H^{s}\left(K_{\delta}\right)}+e^{-\nu T}\left|\Pi_{0} f\right|
\end{aligned}
$$

the second term above is estimated by Proposition 2.1 and the third one tends to zero at $T \rightarrow+\infty$. 
2.2. General case. The idea is to construct an exponentially decaying function $u_{1}$ solving the equation $\square_{g} u_{1}=f$ near the event horizons and then estimate the difference $u-u_{1}$ by Proposition 2.2. We let $u_{1} \in C^{\infty}\left(M_{\delta} \backslash K_{2 \delta}\right)$ solve the following initial/boundary value problem:

$$
\begin{array}{r}
\square_{g} u_{1}=f \text { in } M_{\delta} \backslash K_{2 \delta}, \\
\text { supp } u_{1} \subset\left\{t_{+}>-T\right\} \quad \text { for some } T, \\
\left.u_{1}\right|_{\partial K_{2 \delta}}=0 .
\end{array}
$$

Note that the surfaces $\partial K_{2 \delta}=\left\{r=r_{ \pm} \mp 2 \delta\right\}$ are timelike; therefore, this problem has a unique solution (see, for example, [12, Theorem 24.1.1]. This solution is exponentially decaying in time:

Proposition 2.3. For $\delta>0, \nu>0$ small enough, a small enough depending on $\delta$, and every $s \geq 0$,

$$
\left\|e^{\nu t_{+}} u_{1}\right\|_{H^{s+1}\left(M_{\delta} \backslash K_{2 \delta}\right)} \lesssim\left\|e^{\nu t_{+}} f\right\|_{H^{s}\left(M_{\delta} \backslash K_{2 \delta}\right)} .
$$

Proof. First, consider the case $s=0$. We argue as in the proof of Proposition 1.7, using the vector field $X$ constructed in Proposition 1.6. Namely, we apply the divergence theorem to the vector field $V=e^{2 \nu t_{+}} J_{X}\left(u_{1}\right)$ in the region

$$
\Omega_{T}=\left(M_{\delta} \backslash K_{2 \delta}\right) \cap\left\{t_{+} \leq T\right\} .
$$

The flux of $V$ over $\left\{t_{+}=T\right\}$ and $\partial M_{\delta}$ is nonnegative by Proposition 1.1; the flux over $\partial K_{2 \delta}$ is nonnegative by Proposition 1.2. Computing the divergence of $V$ by Proposition 1.3, we get (2.3).

Now, we assume that $(2.3)$ is true for $s-2$ and prove it for $s$; the rest follows by induction and interpolation in Sobolev spaces. For $a$ small enough, $\partial_{t}$ is timelike in $K_{\delta} \backslash K_{2 \delta}$; therefore, for a large-enough constant $C_{0}$, the operator

$$
L=C_{0} \partial_{t}^{2}-\square_{g}
$$

is elliptic on $K_{\delta} \backslash K_{2 \delta}$. Since $u_{1}$ satisfies the Dirichlet boundary condition on $\partial K_{2 \delta}$, we have

$$
\begin{aligned}
\left\|e^{\nu t_{+}} u_{1}\right\|_{H^{s+1}\left(K_{\delta} \backslash K_{2 \delta}\right)} & \lesssim\left\|e^{\nu t_{+}} L u_{1}\right\|_{H^{s-1}\left(M_{\delta} \backslash K_{2 \delta}\right)}+\left\|e^{\nu t_{+}} u_{1}\right\|_{H^{s-1}\left(M_{\delta} \backslash K_{2 \delta}\right)} \\
& \lesssim\left\|e^{\nu t_{+}} \partial_{t}^{2} u_{1}\right\|_{H^{s-1}\left(M_{\delta} \backslash K_{2 \delta}\right)}+\left\|e^{\nu t_{+}} f\right\|_{H^{s-1}\left(M_{\delta} \backslash K_{2 \delta}\right)} \\
& \lesssim\left\|e^{\nu t_{+}} \partial_{t}^{2} f\right\|_{H^{s-2}\left(M_{\delta} \backslash K_{2 \delta}\right)}+\left\|e^{\nu t_{+}} f\right\|_{H^{s-1}\left(M_{\delta} \backslash K_{2 \delta}\right)} \\
& \lesssim\left\|e^{\nu t_{+}} f\right\|_{H^{s}\left(M_{\delta} \backslash K_{2 \delta}\right)} .
\end{aligned}
$$

Here we applied (2.3) to $u_{1}$ and $\partial_{t}^{2} u_{1}$ and used that $\square_{g}$ commutes with $\partial_{t}^{2}$.

Now, take a nonnegative function $\chi_{\delta}(r) \in C^{\infty}$ such that $\chi_{\delta}=0$ near $K_{2 \delta}$, but $\chi_{\delta}=1$ away from $K_{\delta}$. We can use the above estimate and apply Proposition 1.5 to $\chi_{\delta} u_{1}$ to get

$$
\begin{aligned}
\left\|e^{\nu t_{+}} u_{1}\right\|_{H^{s+1}\left(M_{\delta} \backslash K_{2 \delta}\right) \lesssim} & \left\|e^{\nu t_{+}} u_{1}\right\|_{H^{s+1}\left(K_{\delta} \backslash K_{2 \delta}\right)}+\left\|e^{\nu t_{+}} \chi_{\delta} u_{1}\right\|_{H^{s+1}\left(M_{\delta}\right)} \\
\lesssim & \left\|e^{\nu t_{+}} f\right\|_{H^{s}\left(M_{\delta} \backslash K_{2 \delta}\right)}+\left\|e^{\nu t_{+}} \chi_{\delta} u_{1}\right\|_{H^{s+1}\left(K_{\delta}\right)} \\
& +\left\|e^{\nu t_{+}}\left[\square_{g}, \chi_{\delta}\right] u_{1}\right\|_{H^{s}\left(K_{\delta}\right)} \\
\lesssim & \left\|e^{\nu t_{+}} f\right\|_{H^{s}\left(M_{\delta} \backslash K_{2 \delta}\right)},
\end{aligned}
$$

as required. 
We are now ready to prove Theorem 0.1. Take $\chi_{\delta}$ from the proof of Proposition 2.3 and consider $u_{2}=u-\chi_{\delta}(r) u_{1}$. Then

$$
\square_{g} u_{2}=\left(1-\chi_{\delta}\right) f-\left[\square_{g}, \chi_{\delta}\right] u_{1}
$$

is supported in $K_{\delta}$; moreover, by Proposition 2.3,

$$
\left\|e^{\nu t_{+}} \square_{g} u_{2}\right\|_{H^{s+\varkappa}\left(M_{\delta}\right)} \lesssim\left\|e^{\nu t_{+}} f\right\|_{H^{s+\varkappa}\left(M_{\delta}\right)}
$$

Therefore, we may apply Proposition 2.2 to $u_{2}$ to get

$$
\left\|e^{\nu t_{+}}\left(u_{2}-\Pi_{0}\left(\square_{g} u_{2}\right)\right)\right\|_{H^{s}\left(M_{\delta}\right)} \lesssim\left\|e^{\nu t_{+}} f\right\|_{H^{s+\varkappa}\left(M_{\delta}\right)} .
$$

Note that $\Pi_{0}\left(\square_{g} u_{2}\right)=\Pi_{0} f$, as

$$
\Pi_{0} \square_{g} \chi_{\delta}(r) u_{1}=\lim _{T \rightarrow+\infty} \int_{\partial\left(M \cap\left\{t_{+} \leq T\right\}\right)} g\left(\nabla\left(\chi_{\delta}(r) u_{1}\right), \vec{n}\right) d S .
$$

The integral over the cap $M \cap\left\{t_{+}=T\right\}$ converges to zero, as $u_{1}$ is exponentially decaying in time. As for the timelike piece $\partial M \cap\left\{t_{+} \leq T\right\}$, the normal vector $\vec{n}$ is tangent to $\partial M$ and we can use this to replace the integral of $g\left(\nabla u_{1}, \vec{n}\right)$ over the timelike piece by a certain integral over the spheres $\partial M \cap\left\{t_{+}=T\right\}$; the latter will decay exponentially as $T \rightarrow+\infty$. We now get

$$
\begin{aligned}
\left\|e^{\nu t_{+}}\left(u-\Pi_{0} f\right)\right\|_{H^{s}\left(M_{\delta}\right)} & \lesssim\left\|e^{\nu t_{+}} \chi_{\delta} u_{1}\right\|_{H^{s}\left(M_{\delta}\right)}+\left\|e^{\nu t_{+}}\left(u_{2}-\Pi_{0}\left(\square_{g} u_{2}\right)\right)\right\|_{H^{s}\left(M_{\delta}\right)} \\
& \lesssim\left\|e^{\nu t_{+}} f\right\|_{H^{s+\varkappa}\left(M_{\delta}\right)},
\end{aligned}
$$

which finishes the proof.

\section{Acknowledgments}

I would like to thank Daniel Tataru for suggesting that the results of [10] can lead to improved energy estimates and for providing several key ideas in the proof. Thanks also to Maciej Zworski for helpful discussions and to Kiril Datchev and András Vasy for several enlightening discussions on $[14,9]$. I am also grateful for partial support from NSF grant DMS-0654436. Finally, I am thankful to an anonymous referee for careful reading and suggestions to improve the manuscript.

\section{References}

[1] S. Aretakis, Stability and instability of extreme Reissner-Nordstrm black hole spacetimes for linear scalar perturbations I, Commun. Math. Phys. 307(1) (2011), 17-63, 10.1007/s00220-011-1254-5.

[2] - Stability and instability of extreme Reissner-Nordstrm black hole spacetimes for linear scalar perturbations II, Ann. Henri Poincare (2011), 1-48, 10.1007/s00023-011-0110-7.

[3] J.-F. Bony and D. Häfner, Decay and non-decay of the local energy for the wave equation on the De SitterSchwarzschild metric, Commun. Math. Phys. 282 (2008), 697-719, 10.1007/s00220-008-0553-y.

[4] M. Dafermos, The interior of charged black holes and the problem of uniqueness in general relativity, Commun. Pure Appl. Math. 58(4) (2005), 445-504.

[5] M. Dafermos and I. Rodnianski, The wave equation on Schwarzschild-de Sitter spacetimes, arXiv:0709.2766 (2007).

[6] - Lectures on black holes and linear waves, arXiv e-prints (2008).

[7] - The red-shift effect and radiation decay on black hole spacetimes, Commun. Pure Appl. Math. 62(7) (2009), 859-919.

[8] - A proof of the uniform boundedness of solutions tothe wave equation on slowly rotating Kerr backgrounds, Inven. Math. 185(3) (2011), 467-559, 10.1007/s00222-010-0309-0. 
[9] K. Datchev and A. Vasy, Gluing semiclassical resolvent estimates, or the importance of being microlocal, arXiv:1008.3964 (2010).

[10] S. Dyatlov, Quasi-normal modes and exponential energy decay for the Kerr-de Sitter black hole, Commun. Math. Phys. 306 (2011), 119-163, 10.1007/s00220-011-1286-x.

[11] L. C. Evans and M. Zworski, Semiclassical analysis, in 'Graduate Studies in Mathematics', American Mathematical Society, 2012, Lecture notes available online at http://math.berkeley. edu/ zworski/semiclassical.pdf.

[12] L. Hörmander, The analysis of linear partial differential operators III, Springer, Berlin, 1994.

[13] R. R. Mazzeo and R. B. Melrose, Meromorphic extension of the resolvent on complete spaces with asymptotically constant negative curvature, J. Functional Anal. 75(2) (1987), 260-310.

[14] R. Melrose, A. S. Barreto and A. Vasy, Asymptotics of solutions of the wave equation on de Sitter-Schwarzschild space, arXiv:0811.2229 (2008).

[15] J. V. Ralston, Solutions of the wave equation with localized energy, Commun. Pure Appl. Math. 22(6) (1969), 807-823.

[16] A. Sa Barreto and M. Zworski, Distribution of resonances for spherical black holes, Math. Res. Lett. 4(1) (1997), 103-121.

[17] D. Tataru and M. Tohaneanu, A local energy estimate on Kerr black hole backgrounds, Internat. Math. Res. Notices 2011(2) (2011), 248-292.

[18] M. Taylor, Partial differential equations I. Basic theory, Springer, Berlin, 1996.

[19] J. Wunsch and M. Zworski, Resolvent estimates for normally hyperbolic trapped sets, Ann. Henri Poincare (2011), 1-37, 10.1007/s00023-011-0108-1.

Department of Mathematics, University of California, Evans Hall, Berkeley, CA 94720, USA

E-mail address: dyatlov@math.berkeley.edu 
